\title{
Increase in High-Frequency Magnetic Fields due to Parallel Coils Added to Applicator for Hyperthermia Therapy
}

\author{
S. Yamada, Y. Ikehata, R .Hayashi, T. Ueno, and M. Kakikawa \\ Institute of Nature and Environmental Technology, Kanazawa University,Kanazawa 920-1192, Japan
}

Induction heating type hyperthermia is important area of fusion research in engineering and medicine because of its use as a less invasive cancer therapy. The generation of high-frequency and high-level magnetic fields is a key research issue that will determine the practicality of the medical treatment. We previously proposed double pancake-type coils with wireless transmission. The system generated high-frequency magnetic fields with more than $200 \mathrm{kHz} \times \mathrm{m} T$ on a human scale apparatus. To increase the amplitude of the magnetic field and to decrease losses in exciting coils, we proposed the use of parallel coils that have a special structure consisting of Litz wire. The parallel coils are designed to be connected by wireless transmission instead of a simple parallel connection. This paper discusses the equilibrium of currents on an applicator with wireless transmission that was obtained through simulations and experiments.

Key words: hyperthermia, pancake coil, resonance frequency, parallel coil, wireless transmission.

\section{Introduction}

Induction heating type hyperthermia treatment needs both high magnetic fields with a few hundred $\mathrm{kHz}$ (> $200 \mathrm{kHz} \mathrm{mT}$ ) inside of a body to heat magnetic particles and implant materials up to $43^{\circ} \mathrm{C}^{1), 2)}$. We proposed the double-pancake type coils settled at both side of human body ${ }^{3), 4}$. The exciting coils of human scale could generate magnetic fields with both magnitude and frequency required to a medical treatment. To increase magnetic fields inside a deep body further, we previously proposed that back yoke as magnetic path was added to both coils ${ }^{5)}$. The exciting coil system has a simple structure, but the distribution of magnetic fields is un-uniform and the magnitude decreases in near the centre of coils. When installing the equipment in a therapy room, the flat coil type applicator is considered to be predominance by the physical size and electric power capacity of equipment.

The magnetization coil system has indispensable water-cooling equipment for generating the magnetic fields of a therapeutic level even if the special Litz coil with the developed structure is applied to coil component 2). To improve a resistance loss of coil, an equivalent series-resistance must be decrease absolutely. A number of conductive lines on a Litz wire increases, or the coils of Litz wires are used in parallel. But further increase of conductive lines is unsolvable in the manufacture problem. On the other hand, the parallel coil system brings us the unbalance of currents between parallel coils. We examined the increase of exciting current and the reinforcement of magnetic fields by using parallel coils. But instead of parallel connection, wireless transmission induces currents into parallel coils. The paper discusses the increase of exciting current for higher magnetic fields on an applicator system.

\section{Pancake Coil Type Applicator and Parallel Coils}

\subsection{Configuration of applicator}

An applicator is installed outside of a body to heat magnetic implant and magnetic particles as a thermal source. Fig. 1 shows the double pancake exciting system with two flat spiral coils with 5 turns. Two coils sandwiches a human body from both sides. The distribution of magnetic fields becomes flat and smooth near the center of two coils ${ }^{3), 4)}$. Fig. 2 shows the outline of the exciting system of two coils. We apply a wireless

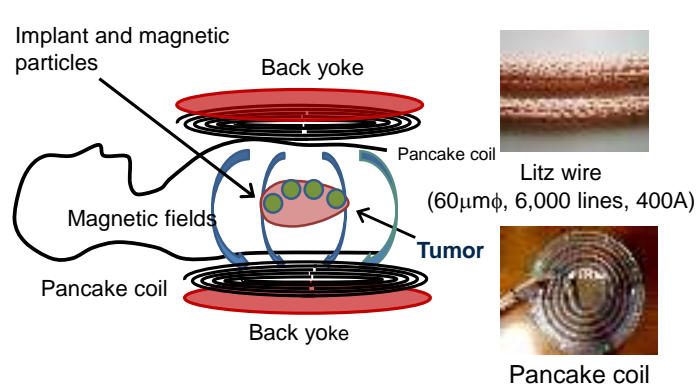

Fig. 1 Induction heating type hyperthermia with double pancake type coils.

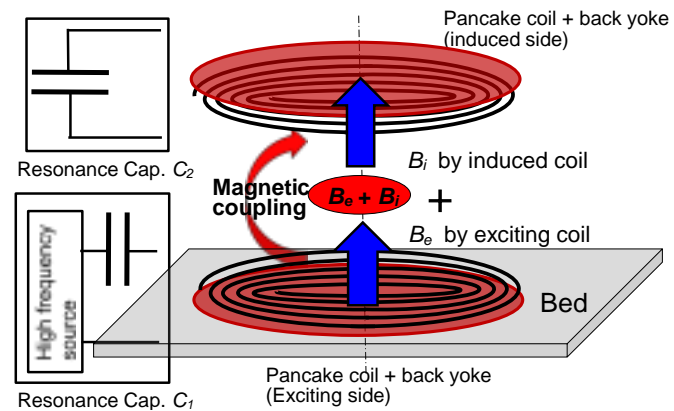

Fig. 2 Applicator system using wireless transmission. 
transmission system to the excitation of double pancake coils. One of pancake coils at the bed side operates as exciting coil and current on the other free coil is induced. The lower coil with series capacitor is connected to a high frequency power source directly and the upper pancake coil is connected to a resonance capacitor. Both coils are connected by magnetic coupling. The previous papers show that the exciting and induced currents have the same amplitude and phase near the resonance frequency ${ }^{3,4}$. Then the magnetic fields by two coils add each other. A back yoke for each coil is settled to increase magnetic fields and to decrease the leakage magnetic field outside of coils ${ }^{5}$.

\subsection{Parallel coil at pancake-type applicator}

To increase the magnetic fields further, it is simple and easy to increase the current capacity of Litz wire. The used Litz wire has the structure like the shielded wire of air-core to consider skin effect at a few hundred $\mathrm{kHz}$ as shown in Fig. 3. But it is difficult to increase a number of lines further(now 6,000 lines). One simple approach is a parallel arrangement by using the present Litz wire. Fig. 3 shows the outline of applicator coils with two parallel wires. Two coils are winded on a plane. Although the inductances $L_{11}, L_{12}$, and $L_{21}, L_{22}$ at the upper and lower coils have the almost same value, the air inductance is not the same at all because air-coil depends on structure. The coupling factor between parallel coils is very near 1 . On the contrary, the coupling between upper and lower is very weak. Then $M$ is much less than $M_{11}$ and $M_{22}$.

\subsection{Connections of parallel coils}

We proposed the connection of parallel wires by wireless transmission like the connection of the upper and lower coils in Fig. 4. One of the coils at the upper side is connected to electric source directly and the induced current is flowed at other coil. Fundamentally inductance $L_{\mathrm{ij}}$, capacitor $C_{\mathrm{ij}}$, and equivalent seriesresistance $r_{\mathrm{ij}}$ are same. On the other hand, a parallel connection of two coils in Fig. 5 is compared with the wireless connection in Fig.4. On the parallel connection, terminals of coils are connected, but inductances are disequilibrium because of manufacturing reason.
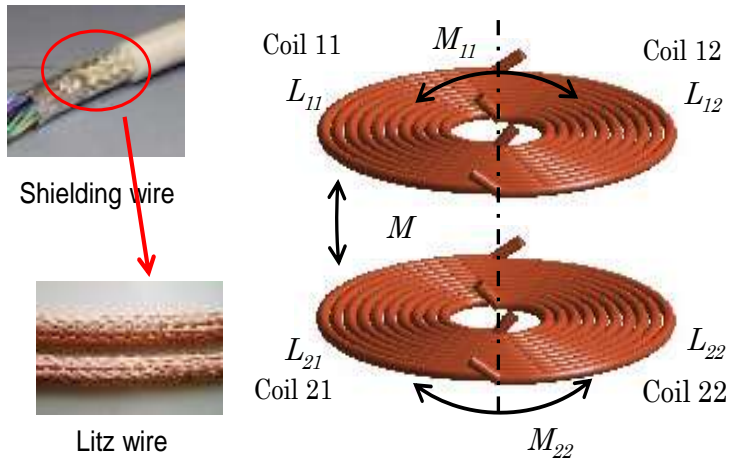

Fig. 3 Litz wire and applicator with parallel coils.

\subsection{Circuit equations and simulation}

To compare the difference of two connections, the circuit equations are formulated by the phasor method ${ }^{3)}$. The circuit equations in Fig. 4 are derived by,

$$
Z_{i} I=V_{i}
$$

where

$$
\begin{aligned}
& \boldsymbol{I}_{i}=\left\{\begin{array}{llll}
\dot{I}_{11} & \dot{I}_{12} & \dot{I}_{21} & \dot{I}_{22}
\end{array}\right\}^{T}, \\
& \boldsymbol{V}_{I}=\left\{\begin{array}{lllll}
V_{i n}^{W} \ldots & O & O & O
\end{array}\right\}^{T}, \\
& Z_{1}=\left[\begin{array}{ccc}
r_{11}+j\left(\omega L_{11}-1 / \omega C_{11}\right) & j \omega M_{11} & -j \omega M \\
j \omega M_{11} & r_{12}+j\left(\omega L_{12}-1 / \omega C_{12}\right) & -j \omega M \\
-j \omega M & -j \omega M & r_{21}+j\left(\omega L_{21}-1 / \omega C_{21}\right) \\
-j \omega M & -j \omega M & j \omega M_{22}
\end{array}{ }^{*}\right. \\
& \left.* \begin{array}{c}
-j \omega M \\
-j \omega M \\
r_{22}+j\left(\omega L_{22}-1 / \omega C_{22}\right)
\end{array}\right]
\end{aligned}
$$

Similarly the impedance matrix $Z_{2}$ in Fig. 5 is given below,

$$
\begin{aligned}
\boldsymbol{V}_{2} & =\left\{\begin{array}{llll}
V_{i n}^{P} & V_{i n}^{P} & 0 & 0
\end{array}\right\}^{T}, \\
Z_{2} & {\left[\begin{array}{ccc}
r_{11}+j\left(\omega L_{11}-1 / \omega C_{1}\right) & j\left(\omega M_{11}-1 / \omega C_{1}\right) & -j \omega M \\
j\left(\omega M_{11}-1 / \omega C_{1}\right) & r_{12}+j\left(\omega L_{12}-1 / \omega C_{1}\right) & -j \omega M \\
-j \omega M & -j \omega M & r_{21}+j\left(\omega L_{21}-1 / \omega C_{2}\right) \\
-j \omega M & -j \omega M & j\left(\omega M_{22}-1 / \omega C_{2}\right)
\end{array}\right.}
\end{aligned}
$$

If $V_{\mathrm{in}} \mathrm{W}=2 V_{\mathrm{in}}^{\mathrm{P}}, \quad C_{1} / 2=C_{11}=C_{12}, \quad C_{2} / 2=C_{21}=C_{22}$, and other circuit parameters are same, the amplitude and phase of

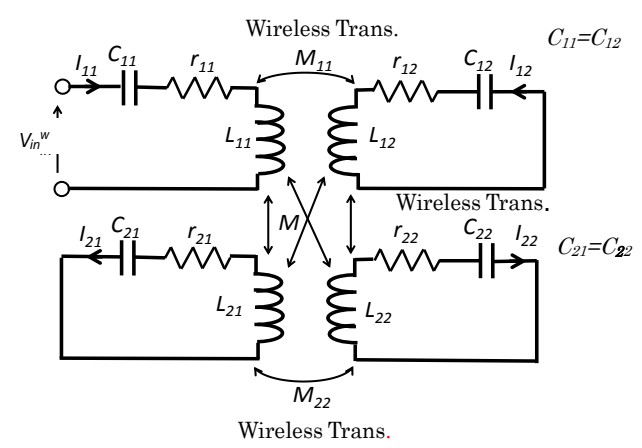

Fig. 4 System with wireless transmission.

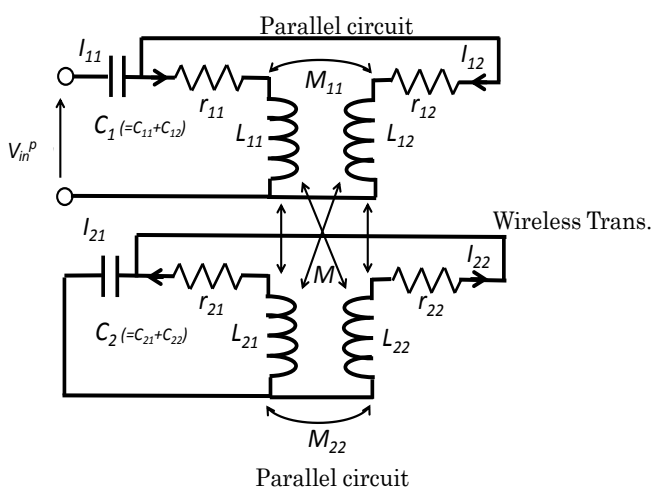

Fig. 5 System with parallel connection. 
all currents are equal at two cases. The simulated results are shown in Fig. 6. The parameters are as follows,

$$
\begin{aligned}
& L_{11}=L_{12}=5.98 \mu \mathrm{H}, L_{21}=L_{22}=5.98 \mu \mathrm{H}, \\
& C_{11}=C_{12}=C_{1} / 2=0.145 \mu \mathrm{F}, \quad C_{21}=C_{22}=C_{2} / 2=0.145 \mu \mathrm{F}, \\
& r_{11}=r_{12}=0.0304 \Omega, \quad r_{21}=r_{22}=0.0195 \Omega \\
& M=0.322 \mu \mathrm{H}, \quad M_{11}=M_{22}=5.74 \mu \mathrm{H}, \\
& V_{\text {in }} \mathrm{W}=V_{\text {in }} \mathrm{P}=2.0 \mathrm{~V} .
\end{aligned}
$$

The currents for parallel connection are twice those for wireless transmission because of the same input voltage.

\section{Balance of Currents in Parallel Coils}

\subsection{Analysis of unbalance of currents}

A few change of circuit constants is not avoided at physical equipment. It is difficult to adjust the self- and mutual-inductances depending on the structure of wires. To evaluate an unbalance of current at parallel coils, one side of double pancake coils is under consideration. Figs. 7 and 8 show

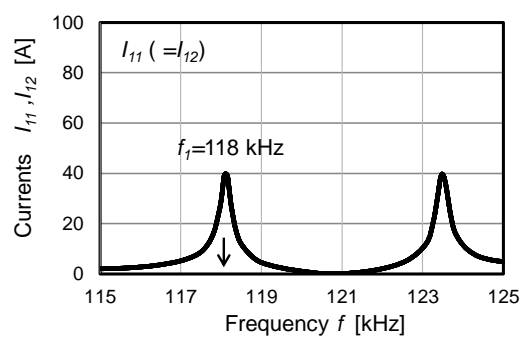

(a) Currents $I_{11}$ and $I_{12}$ for the wireless transmission.

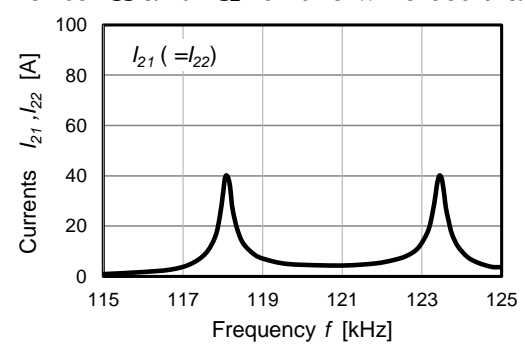

(b) Currents $I_{21}$ and $I_{21}$ for the wireless transmission.

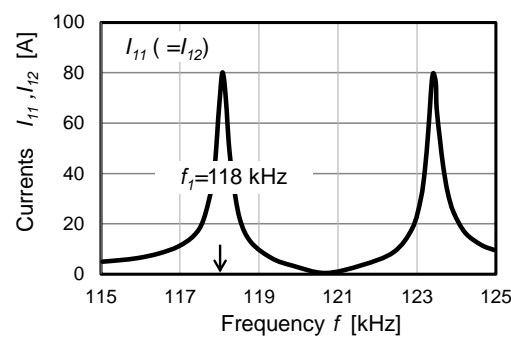

(c) Currents $I_{11}$ and $I_{12}$ for the parallel connection.

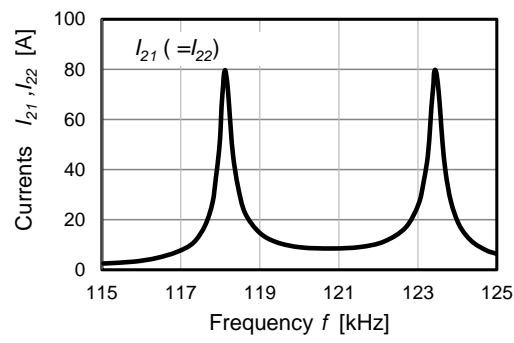

(d) Currents $I_{21}$ and $I_{21}$ for the parallel connection.

Fig. 6 Simulated results for currents. the T-type equivalent circuits of two type connections on the upper side of pancake coils in Figs. 4 and $5^{3)}$. When the currents of parallel coils are $I_{11}$ and $I_{12}$, the ratio of currents for each circuit are given by,

$$
\begin{array}{ll}
\frac{\dot{I}_{12}}{\dot{I}_{11}}=\frac{j \omega M_{11}}{r_{12}+j\left(\omega L_{12}-1 / \omega C_{12}\right)} & \text { (Wireless), } \\
\frac{\dot{I}_{12}}{\dot{I}_{11}}=\frac{r_{11}+j \omega\left(L_{11}-M_{11}\right)}{r_{12}+j \omega\left(L_{12}-M_{11}\right)} & \text { (Parallel). }
\end{array}
$$

When the operation frequency is near the resonance frequency $f_{1}{ }^{3}$, the frequency is given as

$$
f_{1}=\frac{1}{2 \pi \sqrt{C_{12}\left(L_{12}+M_{11}\right)}},
$$

The ratio for wireless connection is given by,

$$
\frac{\dot{I}_{12}}{\dot{I}_{11}}=\frac{j \omega M_{11}}{r_{12}-j \omega M_{11}} \approx-1 \quad\left(r_{12}<<\omega M_{11}\right) \text {. }
$$

The amplitude of the ratio is equal to one. by,

On the contrary, the ratio for wireless connection is

$$
\begin{aligned}
\frac{\dot{I}_{12}}{\dot{I}_{11}}= & \frac{L_{11}-M_{11}}{L_{12}-M_{11}}=\frac{(1-k) L_{0}-\Delta L_{1}}{(1-k) L_{0}-\Delta L_{2}} \\
& \quad\left(r_{11}<<\omega\left(L_{11}-M_{11}\right) \text { and } r_{12}<<\omega\left(L_{12}-M_{11}\right)\right)
\end{aligned}
$$

where the fluctuation of inductances, $L_{11}, L_{12}$, and $M_{11}$ are expressed as,

$$
L_{11}=L_{0}-\Delta L_{1}, L_{12}=L_{0}-\Delta L_{2}, \mathrm{M}_{11}=\mathrm{k} \sqrt{\mathrm{L}_{11} L_{12}} \approx k L_{0} \text {. }
$$

The coefficient $k$ is coupling factor between parallel coils. The value of $k$ is assumed from 0.89 to 0.95 on the measurement. According to Eq. (12), the ratio varies near the conditions $(1-k) L_{0}=\Delta L_{1}$, and $(1-k) L_{0}=\Delta L_{2}$.

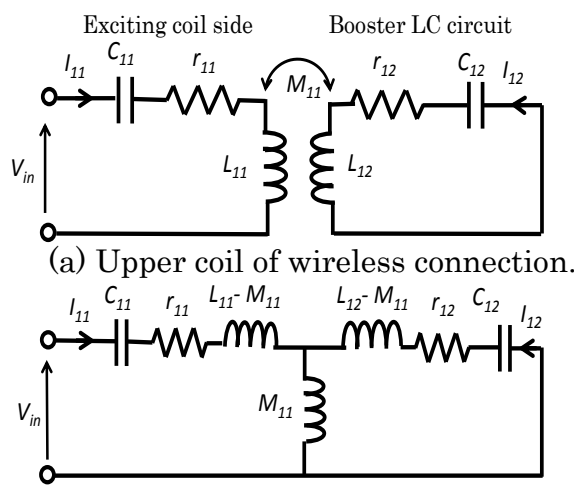

(b) T-type equivalent circuit.

Fig. 7 T-type circuit of wireless connection.

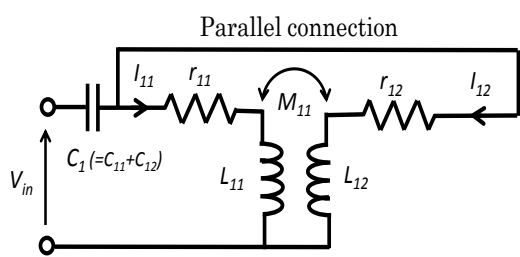

(a) Upper coil of parallel connection.

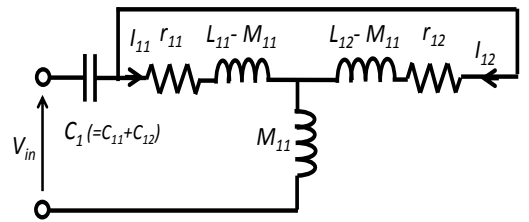

(b) T-type equivalent circuit.

Fig. 8 T- type circuit of parallel connection. 


\subsection{Simulation of unbalance of currents}

We simulated currents by using Eqs.(4) - (6). It is supposed that $\Delta L_{2}$ is zero and $\Delta L_{1}(>0)$ is changed for simplification. When the fluctuation $L_{12} / L_{11}$ and coupling factor $k$ are changed, the ratio $I_{12} / I_{11}$ is drawn on Fig. 9 . For the wireless transmission type, the balance of $I_{12}$ and $I_{11}$ is kept against the change of parameters. On the other hand, for the parallel connection type, the balance of $I_{12}$ and $I_{11}$ is changed much. Then the applicator with transmission system is much better than the other when the parallel coils are used to increase a capacity of currents. Fig. 10 shows the frequency characteristics for two type applicators when the value of inductance $L_{11}$ is changed by $5 \%$. We can observe that the ratio of currents at the coils for exciting side has the clear difference.

\subsection{Experiments results on testing applicator}

We fabricated the testing applicator with the scale suited for human body as shown in Fig. 11. The resonance capacitors are settled outside the pancake coils. The parameters of circuits are listed in Table 1. The capacitors are adjusted as the same value. The gap among two pancake coils is set to be $280 \mathrm{~mm}$. For parallel connection, the difference of inductance is inevitable to connect two wires with only 5 turns. According to the measurement, the disturbance of self-inductances is assumed to be up to $10 \%$.

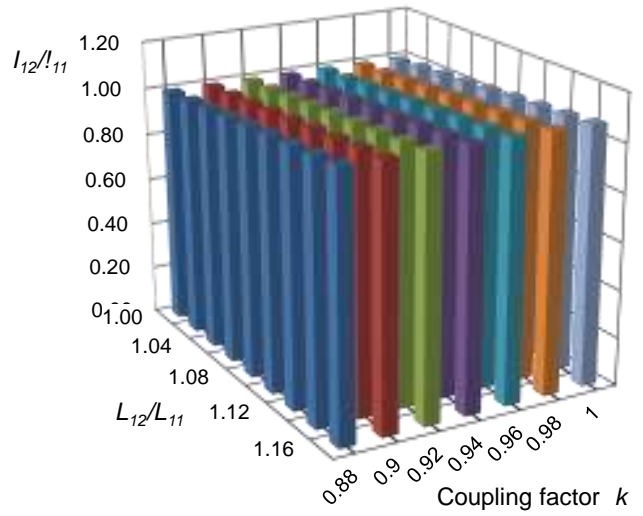

(a) Wireless transmission.

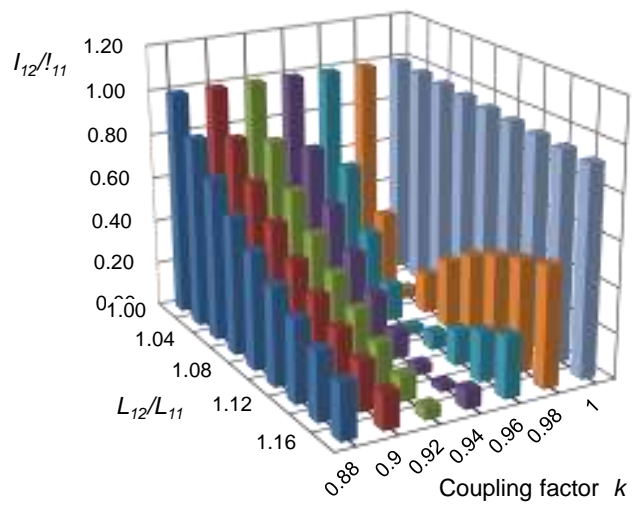

(b) Parallel connection.

Fig. 9 Simulated ratio of currents $I_{11} / I_{12}$.
Fig. 12 shows the comparison of currents $I_{11}$, and $I_{12}$ between two type connections near the resonance frequency $f_{1}$. It is clear that the pancake coil with wireless transmission has the better balance of currents. Fig. 13 shows all amplitudes of currents at 4 coils. We observed that the currents have the good equilibrium in experiments

\section{Conclusion}

The completion of a magnetic field generator with more than $200 \mathrm{kHz} \times \mathrm{mT}$ is indispensable to utilization of induction heating type hyperthermia therapy. We proposed the development of parallel coils connected by wireless transmission to increase magnetic fields. The proposed parallel pancake coils enables us to increase the exciting current capacity keeping the balance of currents in parallel coils.

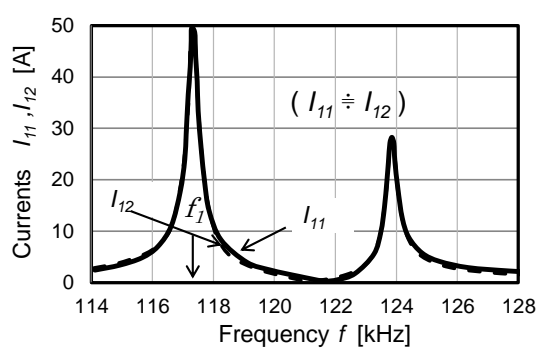

(a) Currents $I_{11}$ and $I_{12}$ for the wireless transmission.

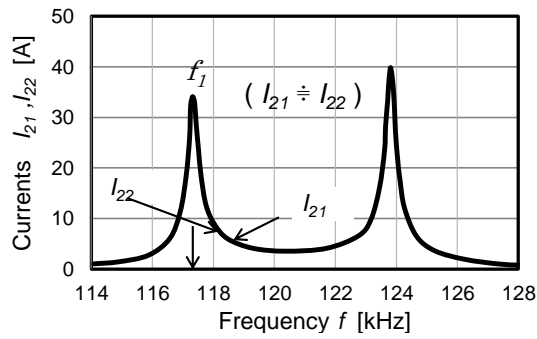

(b) Currents $I_{21}$ and $I_{22}$ for the wireless transmission

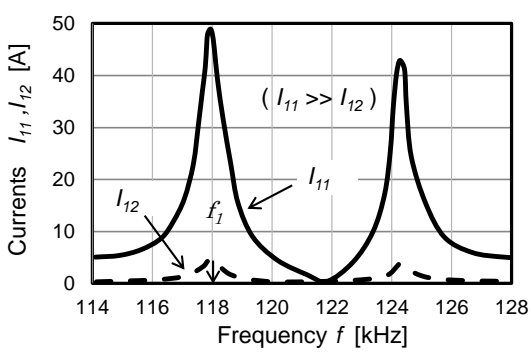

(c) Currents $I_{11}$ and $I_{12}$ for the parallel connection.

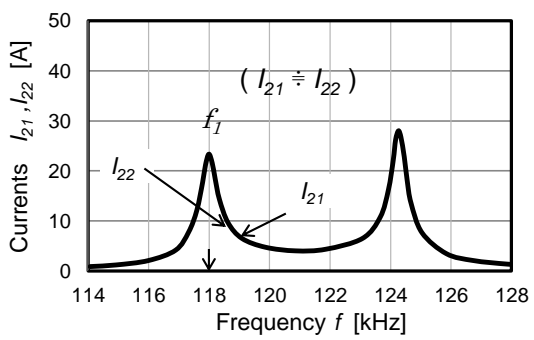

(d) Currents $I_{21}$ and $I_{22}$ for the parallel connection.

Fig. 10 Simulated currents to compare between the parallel connection and the wireless transmission when the value of inductance $L_{11}$ is changed by $5 \%$. 
Table 1 Parameters of equipment

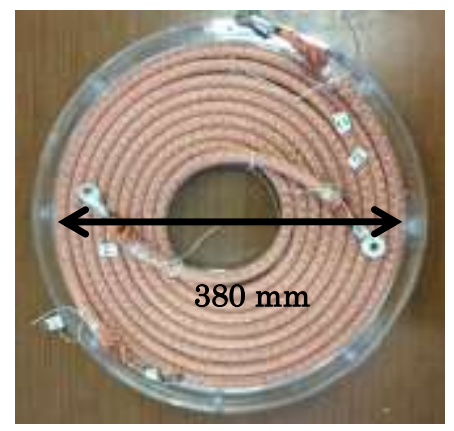

(a) Pancake coil with two parallel Litz wires.
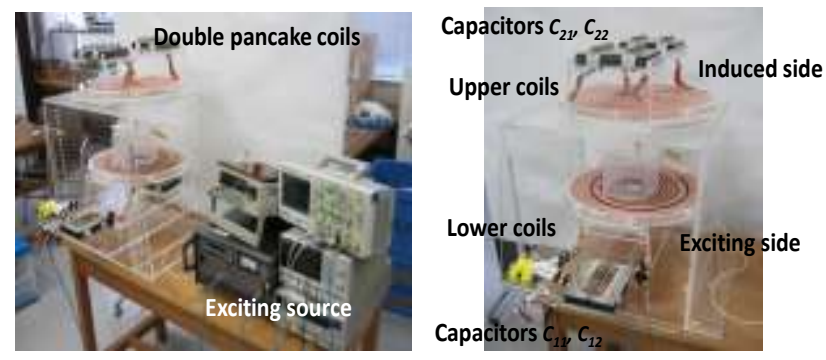

(b) Measuring equipment. (c) Applicator set.

Fig. 11 Testing apparatus.

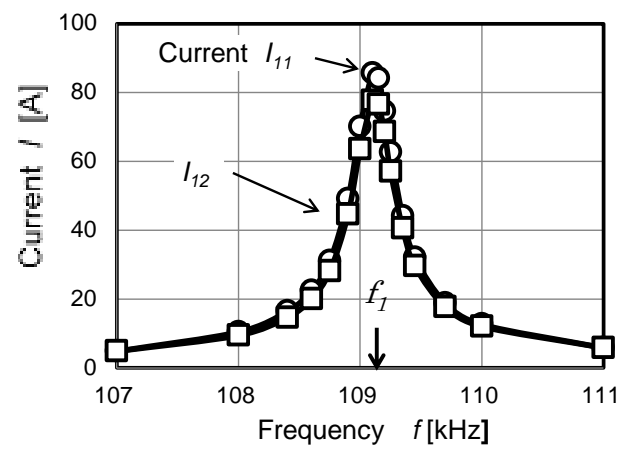

(a) Wireless transmission.

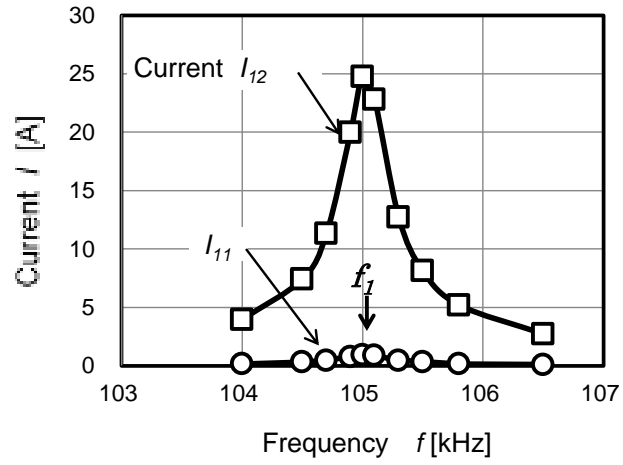

(b) Parallel connection.

Fig. 12 Measured ratio of currents, $I_{11}$, and $I_{12}$ for fluctuations in inductance.

\begin{tabular}{l|l|l}
\hline Element & \multicolumn{1}{|c|}{ Items } & \multicolumn{1}{|c}{ Values \& materials } \\
\hline \multirow{4}{*}{$\begin{array}{l}\text { Pancake } \\
\text { coil }\end{array}$} & Materials & Litz wire $(6,000$ lines $)$ \\
\cline { 2 - 3 } & Parallel coil & Two parallel wires \\
\cline { 2 - 3 } & Diameter & $\begin{array}{l}\text { Outer } 380 \mathrm{~mm} \\
\text { inner } 80 \mathrm{~mm}\end{array}$ \\
\cline { 2 - 3 } & Inductance $L_{\mathrm{ij}}$ & $5.5 \mu \mathrm{H} \pm 10 \%$ \\
\cline { 2 - 3 } & $\begin{array}{l}\text { Equivalent } \\
\text { resistance } R_{\mathrm{s}}\end{array}$ & $4.3 \mathrm{~m} \Omega$ \\
\cline { 2 - 3 } & Coupling factor $k$ & $\begin{array}{l}0.89(\text { Parallel coils }) \\
0.056(\text { Pancake coils } \\
\text { at gap }=280 \mathrm{~mm})\end{array}$ \\
\hline \multirow{2}{*}{ Capacitor } & Materials & Polypropylene \\
\cline { 2 - 3 } & Capacitor $C_{\mathrm{ij}}$ & $220 \mathrm{nF}$ \\
\hline
\end{tabular}

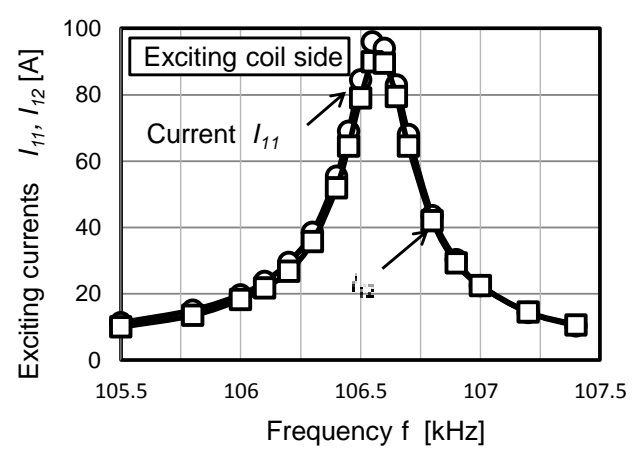

(a) Currents $I_{11}$, and $I_{12}$

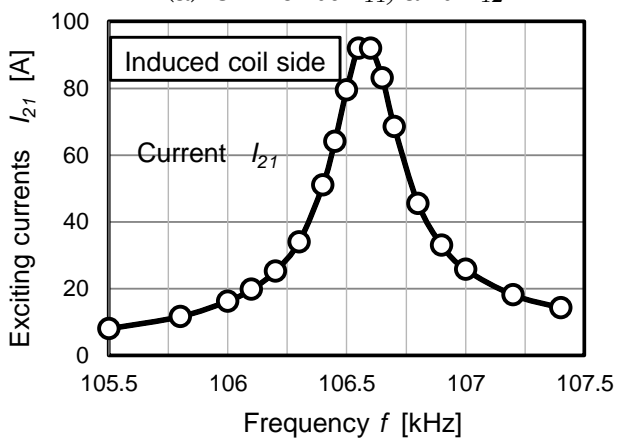

(b) Current $I_{21}$

Fig. 13 Measured currents $I_{11}, I_{12}$, and $I_{21}$ on applicator with wireless transmission.

\section{References}

[1] H.Nagae, S.Yamada, Y.Ikrhata, S.Yagitani, and I.Nagano, IEEJ Trans. on Fundamental and Materials, Vol.133, No. 6, pp.366-371, 2013.

[2] T.Takura, T.Maruyama, F.Sato, H.Matsuki, S.Aiba, and T.Sato, J. Magn. Soc. Jpn., Vol.31, pp.288-292, 2007.

[3] S.Yamada, Y Ikehata, T.Ueno, and M.Kakikawa, J. Magn. Soc. Jpn., Vol.37, pp.282-286, 2013.

[4] S.Yamada, Y Ikehata, T.Ueno, and M.Kakikawa, J. Magn. Soc. Jpn., Vol.38, pp.37-41, 2014.

[5] S.Yamada, Y Ikehata, and R. Hayashi, The paper of Joint Technical Meeting on Magnetics and Linear Drives, IEE Japan, MAG- 14-050, pp.15-18, 2014.[in Japanese]

Received Oct. 07, 2014; Accepted Feb. 02, 2015. 